\title{
Directed growth of horizontal silicon nanowires by laser induced decomposition of silane
}

\author{
H. Abed, ${ }^{\text {a) }}$ A. Charrier, H. Dallaporta, V. Safarov, H. Jamgotchian, and D. Tonneau \\ CRMC-N, Faculté des Sciences de Luminy, Case 913, 13288 Marseille Cedex 09, France
}

We present an original method to force the horizontal growth of silicon nanowires by laser assisted chemical vapor deposition of silane. The $\mathrm{Ar}^{+}$laser beam, tightly focused on the absorbing sample, induces a local thermal horizontal gradient over the laser spot area, which determines the growth direction of the nanowires (NWs). The reaction of formation of $\mathrm{Si}$ NWs occurs via the vapor-liquid-solid process, when gold particles are spread on the surface to catalyze the reaction. The effect of laser power (i.e., of laser induced local temperature) and silane pressure on the morphology of the nanowires is presented.

\section{INTRODUCTION}

Semiconducting nanowires (NWs) present several interesting physical properties among which electrical properties are probably the most important from the point of view of applications (electronic devices, sensors, etc.). The formation of silicon whiskers was observed in a conventional chemical vapor deposition (CVD) reactor from the dissociation of tetrachlorosilane $\left(\mathrm{SiCl}_{4}\right)$ molecules on silicon surface coated with gold clusters. ${ }^{1}$ More recently, the growth of silicon nanowires was studied from the decomposition of silane molecules $\left(\mathrm{SiH}_{4}\right)$, still enhanced by the presence on the surface of gold nanoclusters. ${ }^{2-4}$ The growth of whiskers occurs via the so-called vapor-liquid-solid (VLS) technique. The mechanism involves a step of eutectic $\mathrm{Au}-\mathrm{Si}$ alloy formation at sample temperature higher than $363^{\circ} \mathrm{C}$, i.e., above the cracking temperature of $\mathrm{SiH}_{4}$ molecule $\left(420^{\circ} \mathrm{C}\right)$. The permanent gaseous molecule decomposition induces the oversaturation of the alloy and consequently the precipitation of silicon from the liquid alloy. Finally, one can observe on the sample silicon crystalline NWs, whose diameter is identical to the diameter of the gold clusters. The gold cluster "catalyzing" the gas decomposition remains always at the extremity of the NW during growth. Note that in all these experiments, the whiskers or nanowires grow perpendicularly to the substrate surface, which obliges to develop a heavy process for their integration in a device.

To force the horizontal growth of the nanowire from a gold cluster, we propose to assist the CVD process by a tightly focused laser beam [laser chemical vapor deposition (LCVD)] scanning the sample. The Gaussian beam leads to a local lateral energy density gradient in the surface plane, which will induce the directed growth of the wire from the spot edge to the spot center where the temperature is the highest. ${ }^{5}$ We propose also to address the issue of wire connection to contact leads previously patterned on the oxide sample. Note that Si NWs growth by laser assisted CVD of silane was already observed on the edges of silicon micron-

\footnotetext{
a)Electronic mail: abed@crmen.univ-mrs.fr
}

scale stripes (10-20 $\mu \mathrm{m}$ width), drawn by the laser beam scanning a sample coated with platinum, palladium, or gold thin films, for other applications. ${ }^{6}$

\section{GROWTH TECHNIQUE PRINCIPLE AND EXPERIMENTAL SETUP}

The principle of the growth technique investigated is largely inspired from the VLS technique and presented in Fig. 1. The starting samples are fabricated as described in Sec. III A and consist of bulk silicon templates coated with a thin thermal oxide layer of $200 \mathrm{~nm}$ thickness. A crystalline silicon micronscale test feature is patterned on the sample surface. Then gold clusters are deposited selectively along the edge of the silicon steps dipping the sample in colloidal solutions. Finally, a cw $\mathrm{Ar}^{+}$laser beam $(\lambda=488$ and $514 \mathrm{~nm})$ is tightly focused on the sample surface in the presence of silane $\left(\mathrm{SiH}_{4}\right)$ molecules. The $\mathrm{SiO}_{2}$ layer is transparent to the laser wavelengths which are absorbed by the silicon rear template of the sample. This absorption induces a local surface heating. Assuming a Gaussian shape laser beam, an horizontal thermal gradient appears on the surface, the spot center being the hottest part of the irradiated area. The spot is displaced perpendicularly to the silicon micronics strips, hoping that the local thermal decomposition of silane molecules under the beam and catalyzed by the gold particles will induce the formation of an horizontal Si NW connected to the silicon tracks at both extremities.

Si NWs were synthesized by laser induced CVD of silane (Fig. 2) in the experimental setup described in the previous
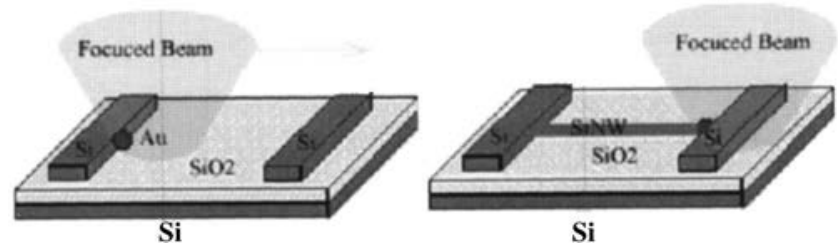

FIG. 1. Schematic representation of silicon lines on the oxide layer with directed growth of silicon nanowires by laser beam. 


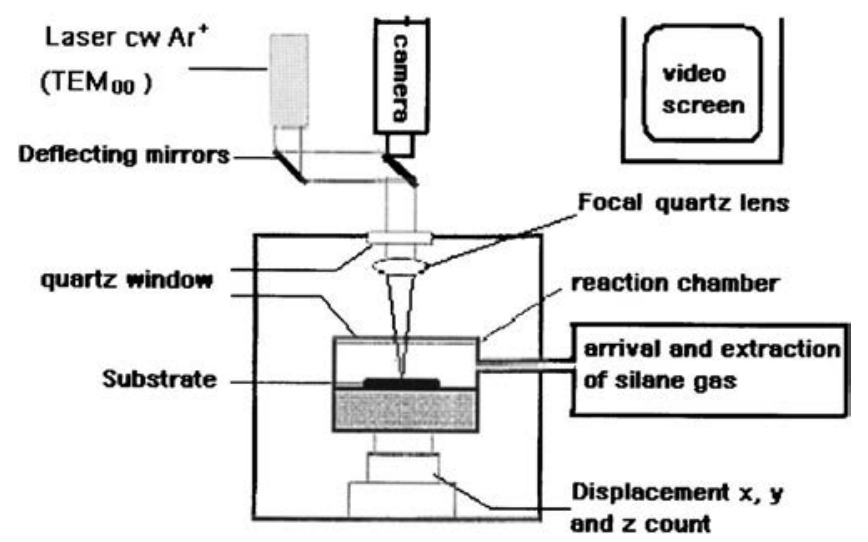

FIG. 2. Schematic diagram of the experimental setup used for Si nanowire growth.

paper. ${ }^{7}$ A chemical chamber of base pressure of $10^{-3}$ mbar is placed on $X, Y, Z$ stages under an optical microscope with three aligned optical paths: two paths for the sample illumination and observation, which forms the optical microscope, and one path for the laser beam. This system allows to tightly focus the laser beam down to a spot diameter of $2.4 \mu \mathrm{m}$ (measured at 1/e) and to observe in real time not only the spot on the sample but also the local chemical reaction while it occurs. The laser is an $\mathrm{Ar}^{+}$laser operating in the Gaussian mode $\mathrm{TEM}_{00}$ and in multiline regime (mainly $514-488 \mathrm{~nm}$ ). The chamber operates in static atmosphere of pure silane and is filled at pressures in the range of 0.1 to 100 mbars as soon as the base pressure is reached. The output laser power was varied between 0.2 and $3 \mathrm{~W}$ and the laser spot can be scanned on the whole sample surface at velocities ranging from 0.1 to $100 \mu \mathrm{m} / \mathrm{s}$.

The silicon nanowires were imaged with a field-emission gun scanning electron microscope (SEM) (JEOL 6320F), associated with an energy dispersive $\mathrm{x}$-ray microanalysis

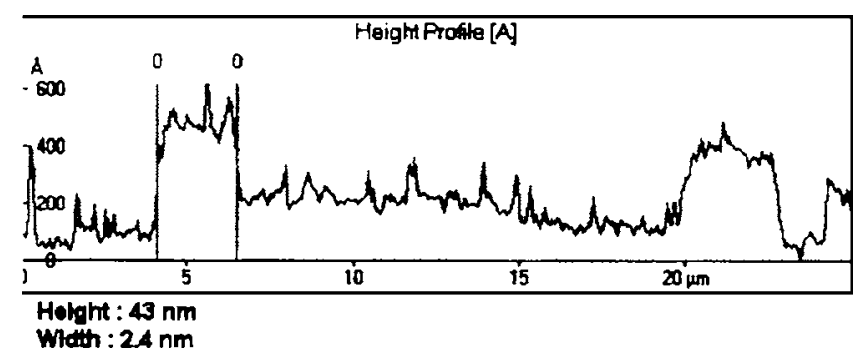

FIG. 4. Cross section of AFM micrograph of silicon lines drawn on the silicon dioxide surface.

(EDX) (Tracor Serie 2). An atomic force microscope (AFM) (Park M5), operating in contact mode, was used to characterize the contact pattern geometry on the oxide coating, before growth experiments.

\section{RESULTS AND DISCUSSION}

\section{A. Sample preparation}

The sample fabrication process is schematically represented in Fig. 3. We started from standard $n$-type $10^{19} \mathrm{~cm}^{-3}$ antimony doped silicon on insulator (SOI) wafers with a silicon top layer thickness of $42 \mathrm{~nm}$. The silicon layer is passivated by hydrogenation in HF solution $(2 \%, 1 \mathrm{~min}$ at room temperature). Silicon oxide strips are then drawn on the sample by local thermal depassivation of the surface under the scanning laser beam operating at high power $(1 \mathrm{~W}$ whereas the laser power threshold to reach the silicon top layer melting is $1.25 \mathrm{~W}$ ) under ambient atmosphere. Finally the sample is dipped in tetramethyl ammonium hydroxide (TMAH) solution at $40{ }^{\circ} \mathrm{C}$ for $2 \mathrm{~min}$, which etches selectively (selectivity of 1:1000) silicon regarding oxide. ${ }^{8}$ This process leads to an insulating surface $\left(\mathrm{SiO}_{2}\right)$, patterned with micronscale heavily doped crystalline contact leads. The

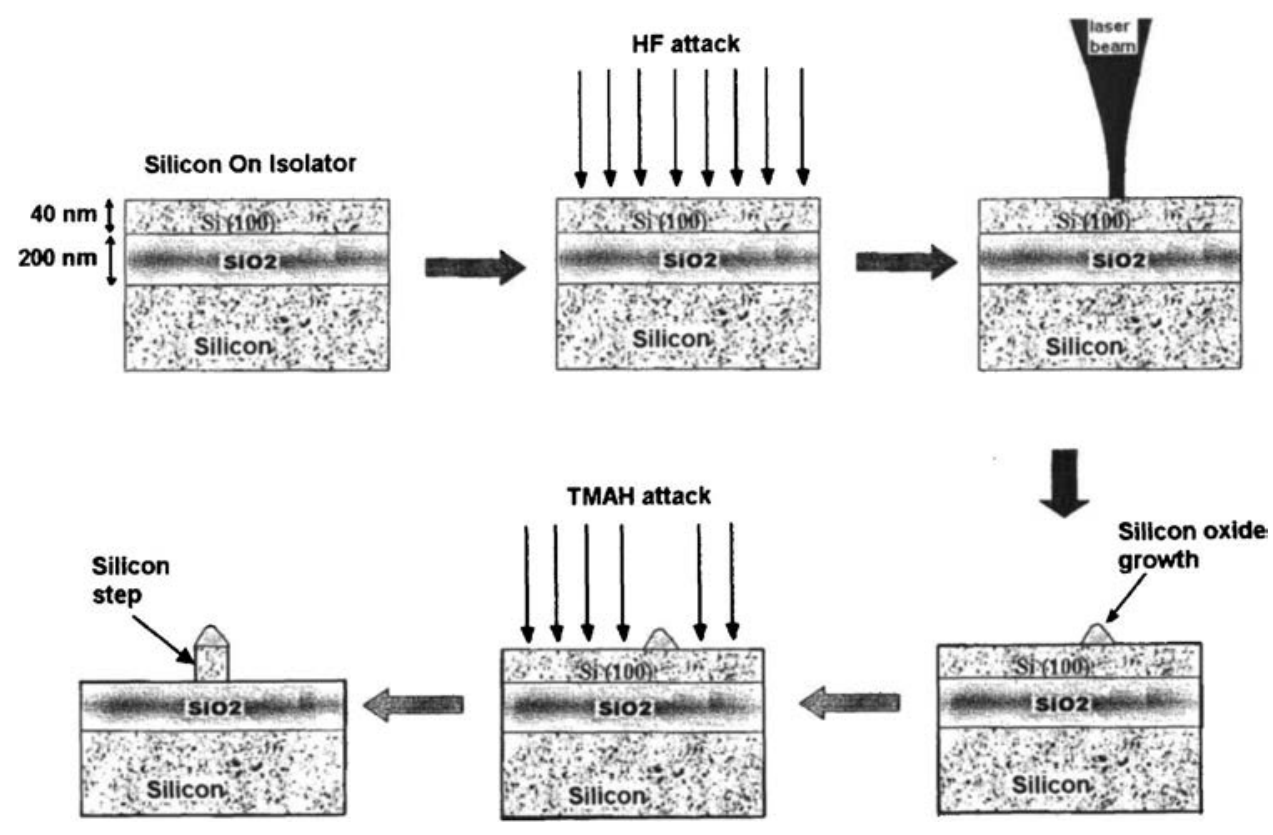

FIG. 3. Schematic sample fabrication process of silicon lines. 


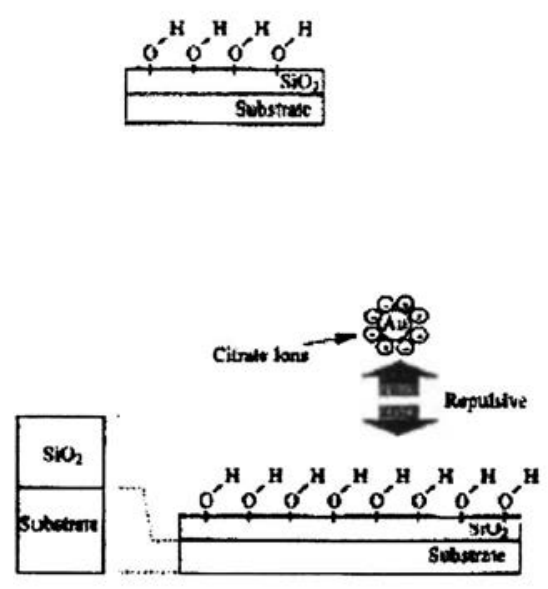

(a)

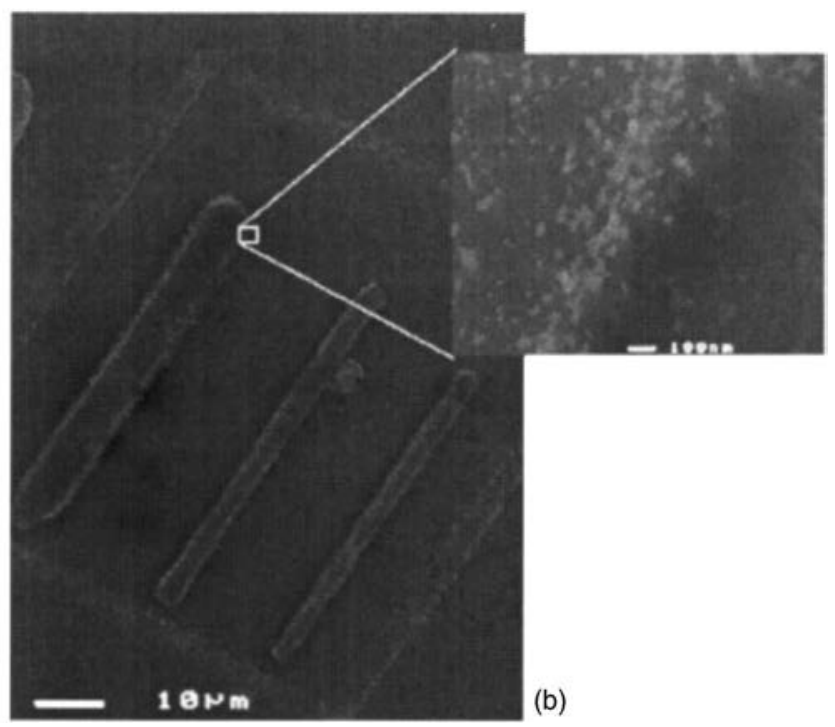

FIG. 5. (a) Scheme of negative citrate Au colloids adsorption on the (b) SEM image of the $\mathrm{SiO}_{2}$ surface patterned by silicon strips after gold colloids adsorption along the silicon walls of the strips.

linewidth is $2.4 \mu \mathrm{m}$, i.e., the laser beam diameter (Fig. 4). The line height of $43 \mathrm{~nm}$ corresponds to the silicon top layer of the starting SOI substrate. These strips remain still coated by the thermal oxide layer drawn by the laser beam [Fig. 3(f)].

\section{B. Gold clusters formation and deposition}

The colloid gold catalyst is synthesized by reduction of $\mathrm{HAuCl}_{4}$ by a solution of sodium citrate. $50 \mathrm{ml}$ of a $1 \mathrm{mM}$ $\mathrm{HAuCl}_{4}$ aqueous solution is first stirred at $110^{\circ} \mathrm{C}$. When reflux is reached, $5 \mathrm{ml}$ of $38.8 \mathrm{mM}$ sodium citrate is added to the solution. The mixture color gradually changes from a light yellow to a ruby red color within 2-3 min marking the production of $15 \mathrm{~nm}$ gold nanoparticles in the solution. Stirring at reflux condition is maintained for $10 \mathrm{~min}$ before slowly cooling down the solution to ambient temperature. ${ }^{9}$ The resulting gold nanoparticles carry a negative surface charge induced by the adsorption of the oxidized negative citrate ions on their surface.

The gold cluster deposition is obtained by dipping the sample into the gold solution for $10 \mathrm{~min}$ before rinsing it in de-ionized (DI) water. Figure 5 shows a tendency for the gold nanoparticles to selectively stick to the edges of the patterns where the silicon is present. This is due to the oxide charge in thermally oxidized $n$-type $\mathrm{Si}$ wafers which is usually negative and that prevents the adsorption of the gold nanoparticles on the silicon oxide areas. ${ }^{10}$

\section{Laser induced local temperature}

One of the main difficulties in laser CVD experiments is the evaluation of the local laser induced temperature within the laser spot area, which is not directly measurable. Consequently, it was calculated by the analytical resolution of the local energy equation. ${ }^{11,12}$ The maximum temperature reached in the center of the spot is given by

$$
T=B+\left(T_{s}-B\right) \exp \left[P\left(1-R_{s}\right) T_{r} / A r_{1 / e}(2 \pi)^{1 / 2}\right],
$$

where $r_{1 / e}$ is the spot radius at $1 / e, P$ the laser beam incidental power, $R_{s}$ the reflectivity of SOI sample after silicon top layer etching, $T_{r}$ the transmission factor through the optical path, $T_{s}$ the substrate temperature (room temperature in our case), and $A$ and $B$ are constants depending on the substrate.

In the case of a silicon sample, $A$ and $B$ coefficients are $A=29900 \mathrm{~W} / \mathrm{m}$ and $B=99 \mathrm{~K}^{11}$ In our case, the sample consists of a silicon wafer coated with a $200 \mathrm{~nm}$ thick silicon dioxide layer. However, we can assume that $A$ and $B$ coefficients are identical Si coefficients, since these parameters are only linked to the thermal properties of the sample, which are not drastically changed by the introduction of a thin silicon dioxide layer whose thickness is ten times smaller than the spot diameter. The transmission factor of the optical path of our LCVD equipment is $T_{r}=0.59$. The calculations do not take into account neither the laser spot scanning speed over the substrate nor the cooling of the heated zone by the ambient gas. In these conditions, we obtain the calibration curve of temperature versus laser power for our sample, $\left(R_{s}=0.5\right)$ under vacuum represented in Fig. 6 (solid line). It was observed that the power threshold for sample melting did not depend on the scan speed in the velocity range investigated. However, when a gas is introduced in the chamber, the laser power needed to melt the sample increases because of the sample cooling by gas thermal conduction. For example, the introduction of nitrogen, whose thermal conduction coefficient is similar to silane (identical molar mass), under a pressure of 5 mbars increases the melting power threshold from 0.9 to $1.3 \mathrm{~W}$. To take into account this important effect of gas pressure, we propose to introduce in Eq. (1) a coefficient $\alpha(p)$ depending on gas pressure and nature as follows: 


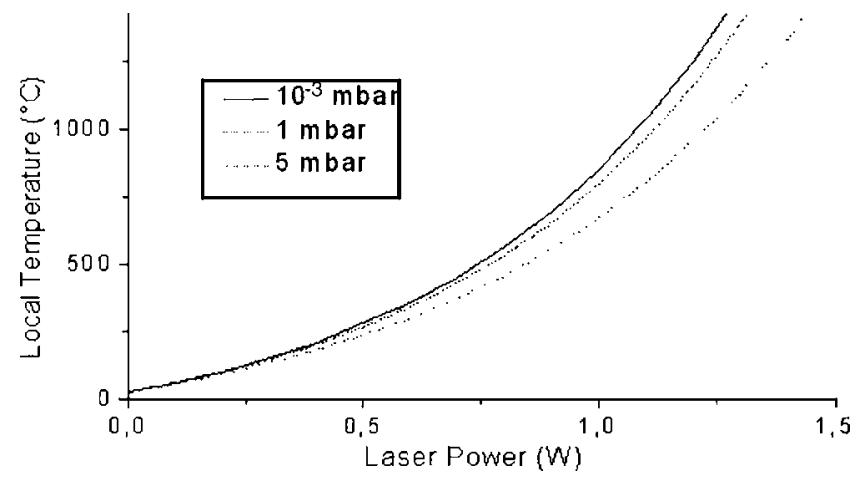

FIG. 6. Maximum temperature reached at the center of the laser spot as a function of the incident laser power at different nitrogen pressures. The substrate is a silicon sample coated by a $200 \mathrm{~nm}$ thick oxide layer.

$$
T=B+\left(T_{s}-B\right) \exp \left[P\left(1-R_{s}\right) T_{r} \alpha(p) / A r_{1 / e}(2 \pi)^{1 / 2}\right],
$$

where $\alpha(p)$ is a term used as a fitting coefficient which is calculated for each gas pressure $p$, knowing the laser power needed to reach the sample melting. Consequently, it is possible to evaluate the local temperature induced by the laser beam as a function of gas pressure (Fig. 6).

\section{Silicon nanowires growth}

A first series of experiments has been carried out on silicon dioxide surfaces obtained by etching the silicon top layer of the SOI sample. The melting power threshold is $1.25 \mathrm{~W}$ under vacuum. In absence of gold clusters, the silane molecules are decomposed above a laser power threshold of $1 \mathrm{~W}$, corresponding to a local maximum temperature of $850{ }^{\circ} \mathrm{C}$ (Fig. 6). This result is in agreement with literature. ${ }^{13,14}$ Figure 7 shows a typical micrometric silicon cluster obtained in these conditions under a silane pressure of 5 mbars, a laser power of $1.1 \mathrm{~W}$, and an irradiation time of $1 \mathrm{~s}$. The diameter of the cluster is about $7 \mu \mathrm{m}$, two to three times the laser spot diameter.

Working on patterned samples, with gold clusters along the silicon steps, the silane decomposition threshold decreases drastically down to $0.16 \mathrm{~W}$, which would induce a local temperature within the laser spot of $100^{\circ} \mathrm{C}$ on a $200 \mathrm{~nm} \mathrm{\textrm {SiO } _ { 2 }}$ layer/Si substrate, under a gas pressure of

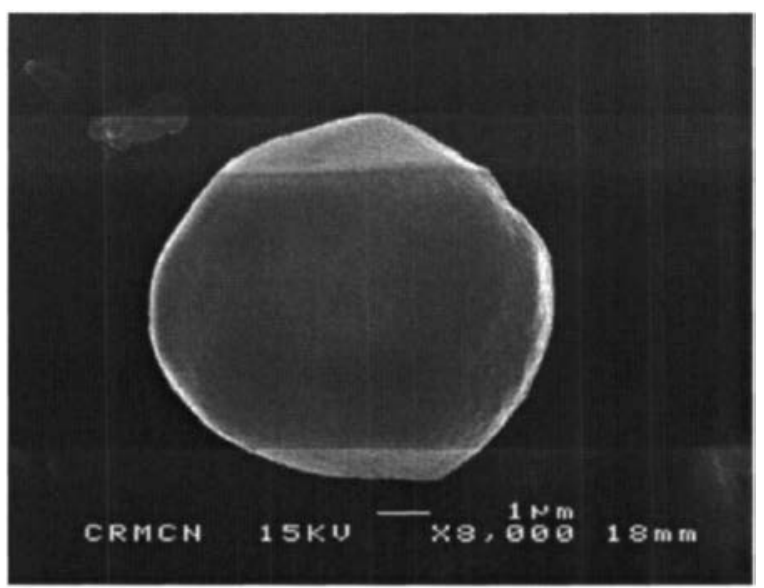

FIG. 7. Silicon feature obtained by decomposition of silane under fixed laser irradiation in absence of colloids, laser power of $1 \mathrm{~W}\left(T=800{ }^{\circ} \mathrm{C}\right)$, silane pressure of 5 mbars, and irradiation time of $1 \mathrm{~s}$.

5 mbars. On the sample we observe the formation of a silicon micronic dot from which start several horizontal silicon nanowires of $15 \mathrm{~nm}$ diameter (diameter of the gold colloids) and 200-300 nm length [Fig. 8(a) and zoom Fig. 8(b)], and one or two bigger nanowires of $200 \mathrm{~nm}$ diameter. Under identical laser power and irradiation time conditions, longer silicon nanowires of $1-2 \mu \mathrm{m}$ can be obtained by increasing the silane pressure up to 100 mbars [Fig. 8(c)].

In literature the formation of silicon nanowires by the VLS technique in a conventional furnace starts above $320{ }^{\circ} \mathrm{C}$ and is usually studied up to $500{ }^{\circ} \mathrm{C}$. ${ }^{15}$ The temperature threshold corresponds to the temperature of eutectic $\mathrm{Si}-\mathrm{Au}$ formation and is from far higher than the threshold of $100{ }^{\circ} \mathrm{C}$ calculated above. This is probably due to the fact that the silicon tracks on the $\mathrm{SiO}_{2} / \mathrm{Si}$ sample absorbs the laser irradiation. Since they lay on a thick $\mathrm{SiO}_{2}$ layer, thermal insulator, their temperature must increase drastically. Consequently, our calculations give a value of local temperature which is undoubtedly underestimated.

EDX analyses have been carried out on the large Si NWs grown on the silicon microdots (Fig. 9). The extremity of the nanowire contains gold [Fig. 9(a)] while the spectra acquired at the bottom of the NW show clearly that the nanowire is

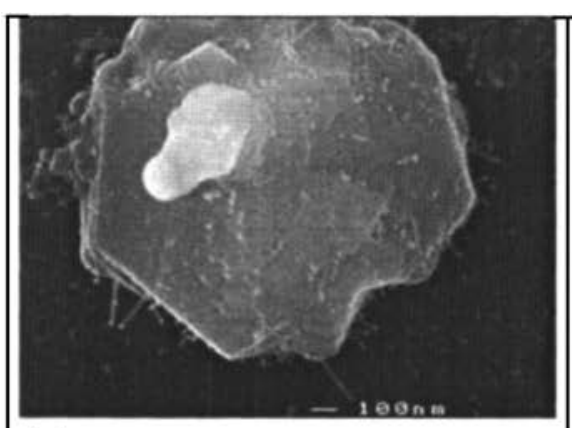

(a)

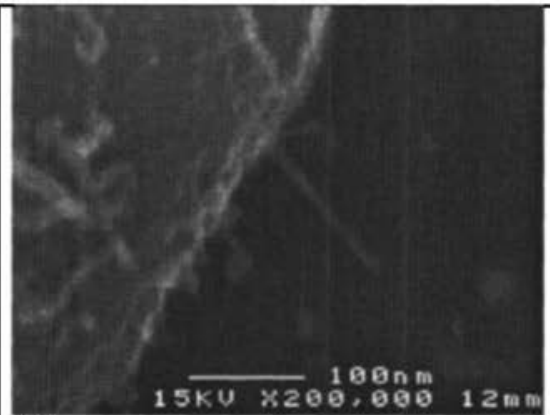

(b)

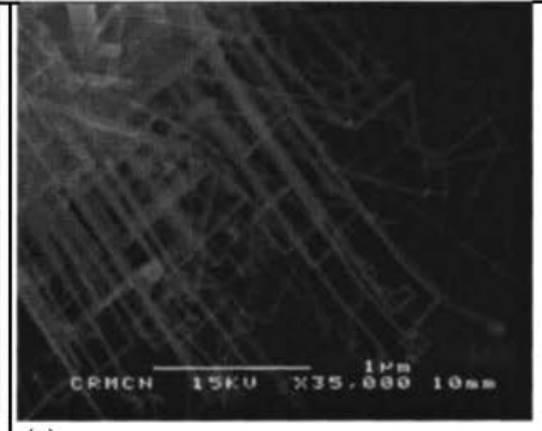

(c)

FIG. 8. (a) SEM analyses of the silicon feature obtained by LCVD of silane in presence of gold colloids (a) laser power of 0.2 W, irradiation time of 1 s, silane pressure of 5 mbars. (b) Zoom on the silicon nanowire. (c) laser power of $0.2 \mathrm{~W}$, irradiation time of $1 \mathrm{~s}$, and silane pressure of 100 mbars. 


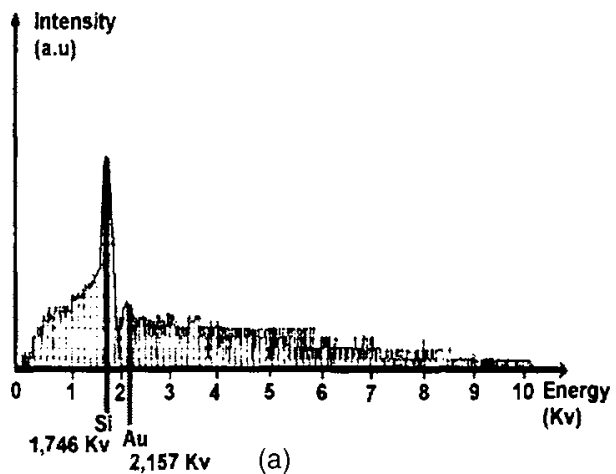

(a)

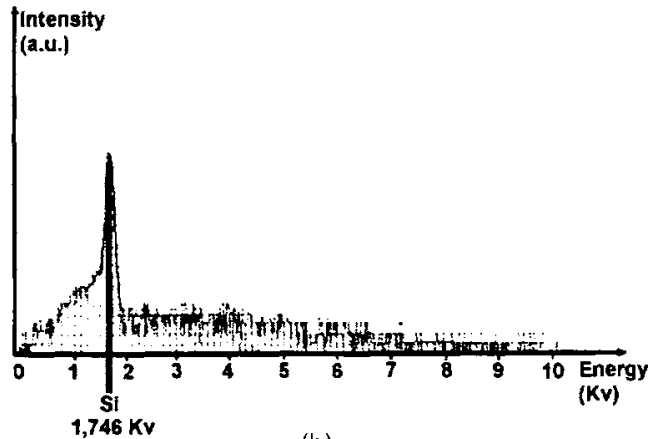

(b)
FIG. 9. EDX analyses of Si NWs. (a) Spectrum of the free extremity of the NW. (b) Typical spectrum at the other extremity of the wire attached to the silicon dot. constituted of pure silicon [Fig. 9(b)]. This observation is in perfect agreement with the expected composition of Si NWs grown by the VLS technique. ${ }^{16,17}$

The next step was to scan the laser beam during the nanowire growth, trying to connect one silicon nanowire. The scan was performed perpendicularly to the silicon leads patterned on the oxide sample (Fig. 10) at a speed of $1 \mu \mathrm{m} / \mathrm{s}$. The lines drawn by the laser beam consist of a series of micronic silicon dots, whose separation depends on the laser scan speed and laser power. It can be easily varied from 0 to $1 \mu \mathrm{m}$.

Figure 11 shows the result of an experience carried out to connect two silicon bumps by a Si NW. In the bottom right of the image, one can see the silicon crystalline step (bright contrast) on the oxide surface (dark contrast). Gold colloids are mainly present along the silicon step. A silicon discontinuous line has been drawn perpendicularly to this contact lead (from bottom right to top left). This line is composed of micronic silicon bumps separated from about $0.5 \mu \mathrm{m}$ apart. We can observe that some Si NWs start only on the bump directly linked to the crystalline silicon leads, i.e., on the only area where gold colloids are present. No Si NWs are

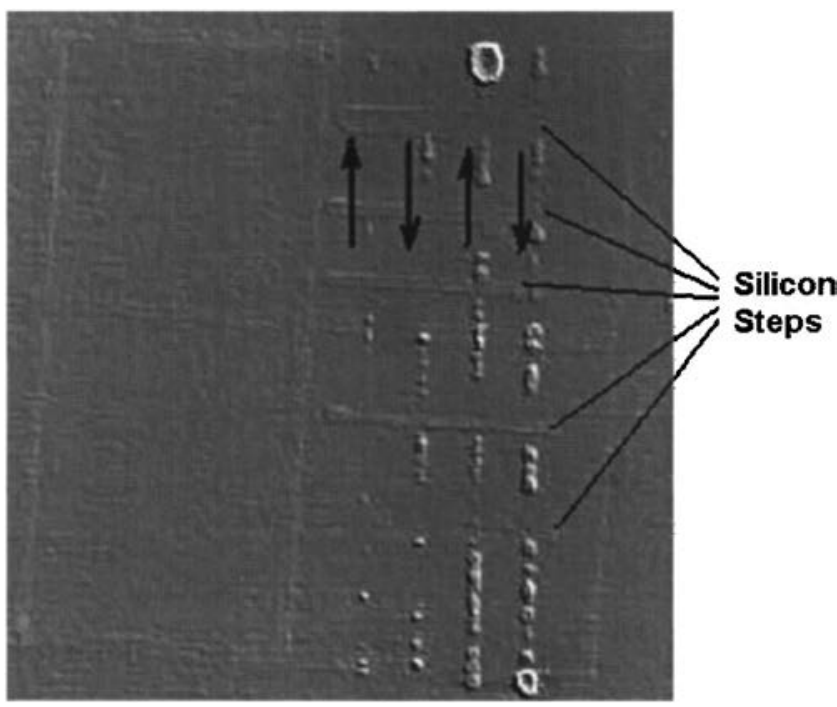

FIG. 10. SEM micrograph of silicon lines drawn between the silicon leads by the laser beam by local decomposition of silane molecules. The silane pressure is 5 mbars and the laser power varies from 0.16 to $0.22 \mathrm{~W}$ by steps of $0.02 \mathrm{~W}$. The laser scan speed was $1 \mu \mathrm{m} / \mathrm{s}$. observed on the next bump, grown on silicon oxide layer, i.e., in absence of colloids. Furthermore the NW growth is directed along the laser beam scan axis, which is a very promising result to obtain connected Si NWs.

\section{CONCLUSION}

Horizontal directed growth of silicon nanowires is possible by laser assisted CVD of silane on silicon surfaces coated with gold colloids. The reaction of NWs formation is catalyzed by the presence of gold particles and occurs above a temperature threshold below the cracking temperature of silane in a CVD furnace $\left(420{ }^{\circ} \mathrm{C}\right)$. This result is consistent with the temperature threshold of $\mathrm{Si}$ NWs formation of $360{ }^{\circ} \mathrm{C}$ observed in a furnace. The diameter of the wires is the same as the gold particle's diameter (15 $\mathrm{nm}$ in our case). The length of the wire can be varied between 200 and $1 \mu \mathrm{m}$ by varying the silane pressure in the chamber, under given laser power and irradiation time conditions.

Si NWs have one extremity connected to a silicon bump, and we can reasonably hope to connect both extremities of the wire to silicon microdots by scanning the laser beam on the surface. Thus this process seems to be of high interest to fabricate connected Si NWs, which would allow their integration in a device, such as a sensor. Furthermore, adding $\mathrm{BCl}_{3}$ or $\mathrm{PH}_{3}$ gas to the silane gas phase, doped wires could

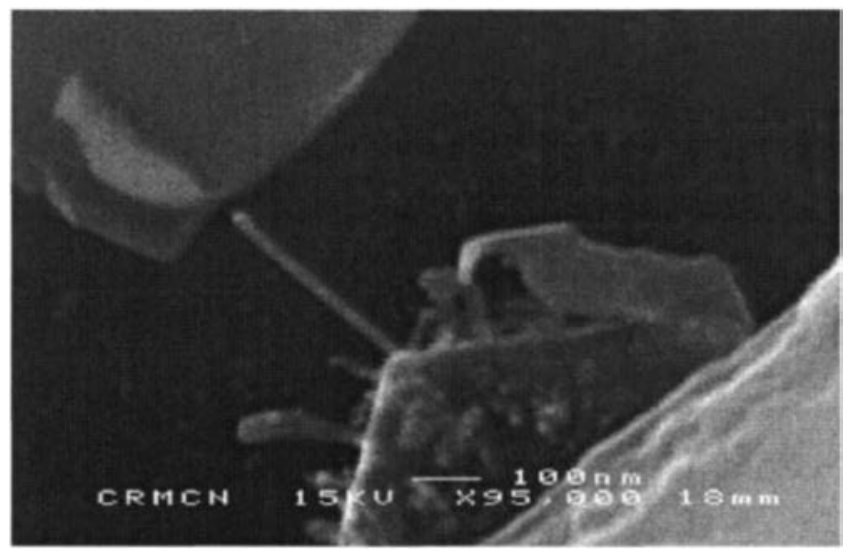

FIG. 11. Si NW linked at one extremity to a silicon bump deposited on the edge of a silicon step. Its other extremity is directed to the following silicon bump. Experimental conditions: laser power of $0.18 \mathrm{~W}$, silane pressure of 5 mbars, and laser scan speed of $1 \mu \mathrm{m} / \mathrm{s}$. 
be easily obtained, and $P N$ junctions could be fabricated, by switching the gaseous phase during the wire growth. Finally, the process proposed would allow also the in situ encapsulation of the nanowire by a thermal oxide growth after NWs formation.

Of course, the present process cannot lead to the precise positioning of a single gold cluster at the extremity of one contact lead. This location problem will be addressed in a further step, starting with identical patterned samples whose contact lead extremities will be decorated by one gold cluster deposited by e-beam CVD of gold precursors. ${ }^{18}$ Concerning the growth direction, it has already been demonstrated that it can be forced in a given direction under application of an electric field, ${ }^{19}$ which can be easily achieved with the patterned sample.

\footnotetext{
${ }^{1}$ R. S. Wagner, J. Appl. Phys. 38, 1554 (1966).

${ }^{2}$ A.-M. Morales and C. Lieber, Science 279, 208 (1998).

${ }^{3}$ E. W. Wong, P. E. Sheedan, and C. Lieber, Science 277, 1971 (1997).

${ }^{4}$ R. Rao et al., MRS Symposia Proceedings No. 737 (Materials Research
}

Society, Pittsburgh, 2002).

${ }^{5}$ G. Pelous, Y. Guern, D. Gobleid, J. David, A. Chion, and D. Tonneau, Proc. SPIE 1598, 149 (1991).

${ }^{6}$ P. C. Nordine, S. C. Delaveaux, and F. T. Wallenberger, Appl. Phys. A: Solids Surf. 57, 97-100 (1993).

${ }^{7}$ D. Tonneau, G. Auvert, and Y. Pauleau, Thin Solid Films 155, 75 (1987).

${ }^{8}$ F. S. S. Chien, W. F. Hsieh, S. Gwo, A. E. Vladar, and J. A. Dagata, J. Appl. Phys. 91, 10045 (2002).

${ }^{9}$ J. J. Storhoff, R. E. Elghanian, R. C. Mucic, C. A. Mirkin, and R. L. Letsinger, J. Am. Chem. Soc. 120, 1959 (1998).

${ }^{10}$ C. Munakata and H. Shimizu, Semicond. Sci. Technol. 15, 40 (2000).

${ }^{11}$ Y. I. Nissim, A. Lietoila, R. B. Gold, and J. F. Gibbons, J. Appl. Phys. 51, 274 (1979)

${ }^{12}$ J. E. Moody and R. H. Hendel, J. Appl. Phys. 53, 4364 (1982).

${ }^{13}$ G. Auvert, D. Tonneau, and Y. Pauleau, Appl. Phys. Lett. 52 (1988).

${ }^{14}$ D. Tonneau, G. Auvert, and Y. Pauleau, J. Vac. Sci. Technol. A 4, 670 (1986).

${ }^{15}$ J. Westwater, D. P. Gosain, S. Tomiya, S. Usui, and H. Ruda, J. Vac. Sci. Technol. B 15, 554 (1997).

${ }^{16}$ N. Ozaki, Y. Ohno, and S. Takeda, Appl. Phys. Lett. 73, 3700 (1998).

${ }^{17}$ G. A. Bootsma and H. J. Gassen, J. Cryst. Growth 10, 223 (1971).

${ }^{18}$ T. Brintlinger et al., J. Vac. Sci. Technol. B 23, 3174 (2005).

${ }^{19}$ O. Englander, D. Chrstensen, and L. Liwei Lin, Appl. Phys. Lett. 82, 4797 (2003). 